\title{
Structural Identification of Crystal Lattices Based On Fuzzy Neural Network Approach
}

\author{
Dmitriy Kirsh \\ Samara University, \\ IPSI - Branch of the FSRC \\ "Crystallography and Photonics" \\ of RAS \\ 443001, Samara, Russia \\ kirshdv@gmail.com

\section{Olga Soldatova} \\ Samara University, \\ 443001, Samara, Russia \\ op-soldatova@yandex.ru
}

\author{
Alexandr Kupriyanov \\ Samara University, \\ IPSI - Branch of the FSRC \\ "Crystallography and Photonics" \\ of RAS \\ 443001, Samara, Russia \\ alexkupr@gmail.com
}

Ilya Lyozin

Samara University,

443001, Samara, Russia

ilyozin@yandex.ru

\author{
Rustam Paringer \\ Samara University, \\ IPSI - Branch of the FSRC \\ "Crystallography and Photonics" \\ of RAS \\ 443001, Samara, Russia \\ rusparinger@gmail.com
}

\author{
Irina Lyozina \\ Samara University, \\ 443001, Samara, Russia \\ chuchyck@yandex.ru
}

\begin{abstract}
Each crystal nanostructure consists of a set of minimal building blocks (unit cells) which parameters comprehensively describe the location of atoms or atom groups in a crystal. However, structure recognition is greatly complicated by the ambiguity of unit cell choice. To solve the problem, we propose a new approach to structural identification of crystal lattices based on fuzzy neural networks. The paper deals with the TakagiSugeno-Kang model of fuzzy neural networks. Moreover, a three-stage neural network learning process is presented: in the first two stages crystal lattices are grouped in non-overlapping classes, and lattices belonging to overlapping classes are recognized at the third stage. The proposed approach to structural identification of crystal lattices has shown promising results in delimiting adjacent lattice types. The structure identification failure rates decreased to $10 \%$ on average.
\end{abstract}

\section{Keywords}

crystal lattice, fuzzy neural networks, crystal structure identification, lattice system, unit cell, Takagi-Sugeno-Kang neural network, Wang-Mendel neural network.

\section{INTRODUCTION}

Being the fundamental concept of crystallography and having Angstrom-order sizes, Bravais lattices are building blocks for all crystals. Every crystal is constructed of these lattices in various modifications. At the same time, different crystals can have the same lattices. There is a total of 14 such lattices. Depending on special symmetry, all crystals are distributed among seven lattice systems: triclinic, monoclinic, tetragonal, orthorhombic, trigonal, hexagonal, and cubic systems [Til01a]. Figure 1 presents the general arrangements of Bravais lattices (smallest structural blocks) for each lattice system.

Permission to make digital or hard copies of all or part of this work for personal or classroom use is granted without fee provided that copies are not made or distributed for profit or commercial advantage and that copies bear this notice and the full citation on the first page. To copy otherwise, or republish, to post on servers or to redistribute to lists, requires prior specific permission and/or a fee.
The type of a lattice system is determined by six parameters of a Bravais lattice: the lengths of the three edges and three angles between them (Fig. 2) [Kup01a]. Table 1 shows properties of the lattice systems.

The task of recognizing nano-scale images, which are projections of crystal lattices, can be reduced to the structure identification problem. However, the major difficulty is the ambiguity in choosing a twodimensional basic cell for a particular projection (Fig. 3) [Ham01a].

Since the classes of Bravais lattices are overlapping, our idea is to use fuzzy neural networks. This kind of networks combines learning and generalization abilities of neural nets, fuzzy logic operations (which allow us to determine the degree of class inclusion of an object as a real number from 0 to 1 ), and possibility to classify fuzzy rule-oriented bases. A class with the highest degree of class inclusion is the result of structure identification. 

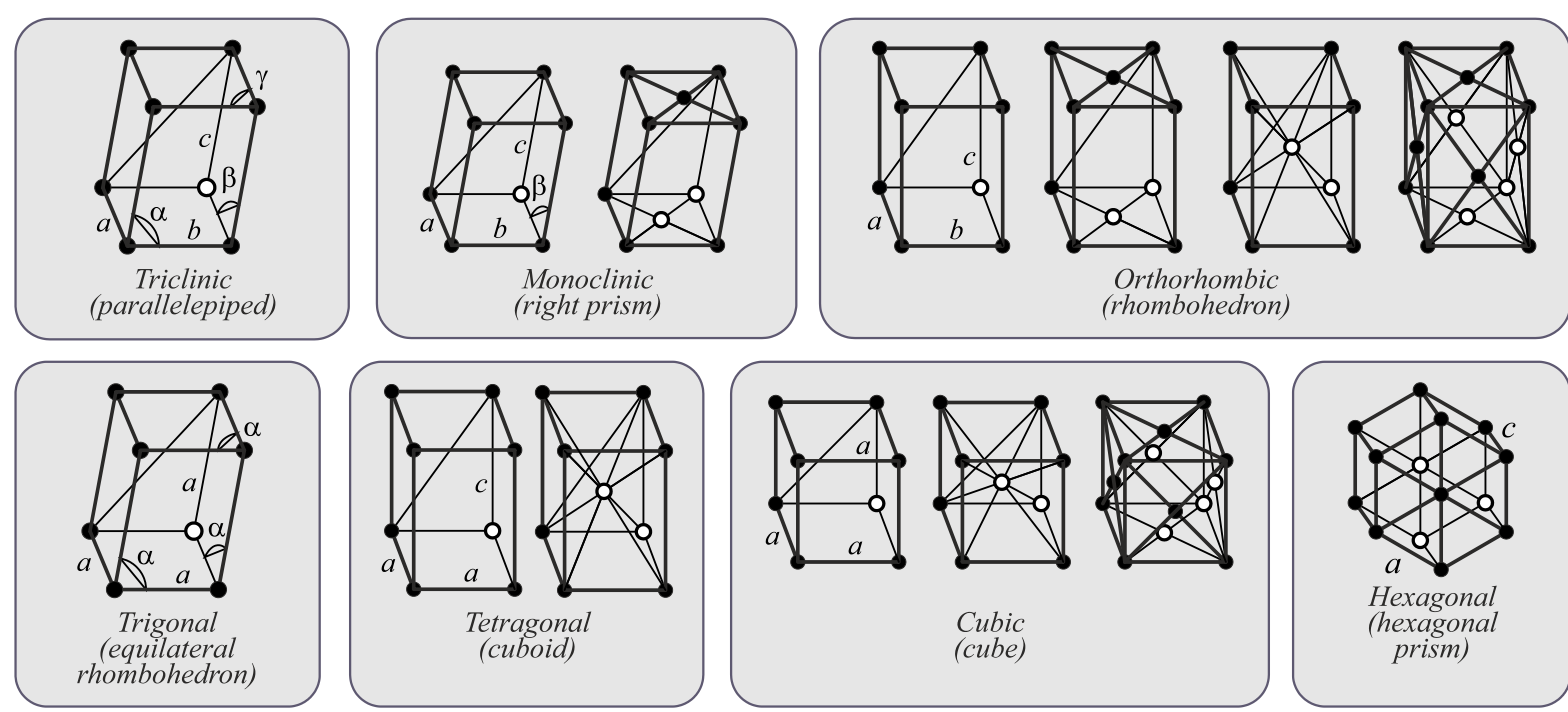

Figure 1. The unit cells of seven lattice systems.

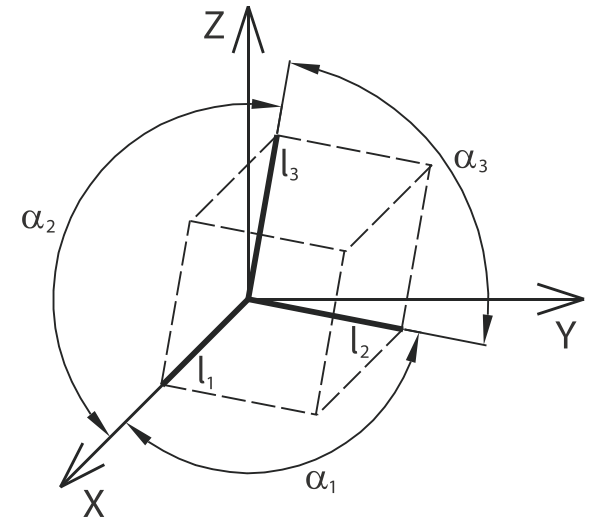

Figure 2. The main parameters of a Bravais unit cell.

\begin{tabular}{|l|c|c|l|}
\hline \multicolumn{1}{|c|}{$\begin{array}{c}\text { Lattice } \\
\text { system }\end{array}$} & Sym. & \multicolumn{1}{c|}{ Edges } & \multicolumn{1}{|c|}{ Angles } \\
\hline Triclinic & $a P$ & $l_{1} \neq l_{2} \neq l_{3}$ & $\alpha_{1} \neq \alpha_{2} \neq \alpha_{3}$ \\
\hline Monoclinic & $m P$ & $l_{1} \neq l_{2} \neq l_{3}$ & $\alpha_{1}=\alpha_{2}=90^{\circ} \neq \alpha_{3}$ \\
\hline Orthorhombic & $o P$ & $l_{1} \neq l_{2} \neq l_{3}$ & $\alpha_{1}=\alpha_{2}=\alpha_{3}=90^{\circ}$ \\
\hline Tetragonal & $t P$ & $l_{1}=l_{2} \neq l_{3}$ & $\alpha_{1}=\alpha_{2}=\alpha_{3}=90^{\circ}$ \\
\hline Cubic & $c P$ & $l_{1}=l_{2}=l_{3}$ & $\alpha_{1}=\alpha_{2}=\alpha_{3}=90^{\circ}$ \\
\hline Trigonal & $h R$ & $l_{1}=l_{2}=l_{3}$ & $\alpha_{1}=\alpha_{2}=\alpha_{3} \neq 90^{\circ}$ \\
\hline Hexagonal & $h P$ & $l_{1}=l_{2} \neq l_{3}$ & $\begin{array}{l}\alpha_{1}=120^{\circ} ; \\
\alpha_{2}=\alpha_{3}=90^{\circ}\end{array}$ \\
\hline
\end{tabular}

Table 1. Lattice systems properties.

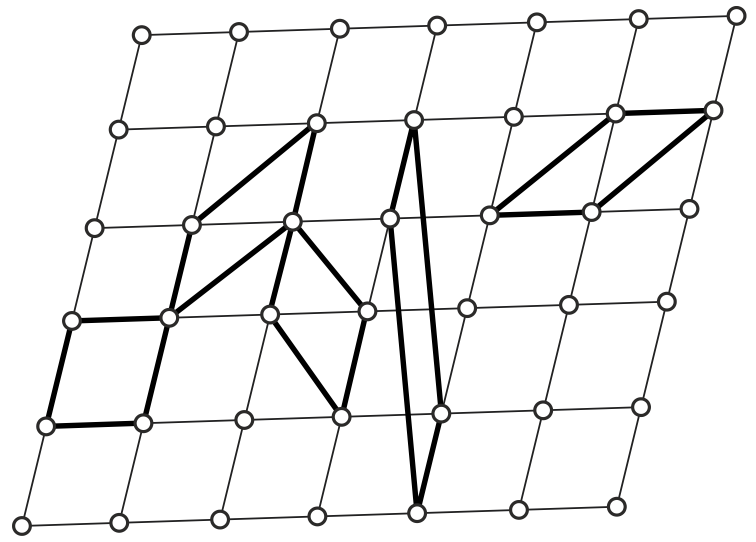

Figure 3. Ambiguity of unit cell choice for a two-dimensional basic cell.

The determination of classification parameters from experimental results and expert evaluation of the parameters are the most popular classification methods using fuzzy neural nets. Particularly, the author of paper [Vin01a] modifies Takagi-SugenoKang (TSK) neural network by introducing the recurrent TSK net. The trick allows the automatic generation of fuzzy rules, but increases the computational complexity of the learning algorithm. Paper [Kip01a] proposes a fuzzy TSK neural network for tackling the classification problem. The net uses the expert evaluation method to choose the most informative classification features and form fuzzy inference rules. In paper [Kat01a] similar approaches are used for learning the author's modification of the Wang-Mendel network. The drawback of the method is the use of subjective estimations of fairly large number of experts and necessity to evaluate their consistency.

Conventional fuzzy rule-based neural net models and modified TSK network use the algebraic product or 
minimum-form logical product as a fuzzy Boolean conjunction. Respectively, these models use algebraic sum or maximum-form Boolean sum as a fuzzy Boolean disjunction [Kat01a, Oso01a, Rut01a, Vin01a]. At the same time research [Nov01a] allows a conclusion about the effective use of fuzzy logical operations used in algebras of Goedel, Goguen and Lukasiewicz. Paper [Sol01a] offers and investigates modifications of Wang-Mendel networks that allows us to operate fuzzy logical operations defined in these algebras.

The paper is aimed at solving the crucial problem of ambiguity of unit cell choice that greatly decrease the quality of crystal structure recognition. We propose a new approach to structural identification of crystal lattices based on fuzzy neural network. In particular, the fuzzy TSK neural network model has been investigated using a sample of 7000 parameter sets of Bravais lattices belonging to 7 lattice system classes.

This paper is organized in the following way. At first, existing parametric identification approaches are described. Afterwards, we will explain the proposed fuzzy network model and learning technique. The last two sections are devoted to the identification method comparisons, error analysis and conclusions.

\section{PARAMETRIC IDENTIFICATION APPROACHES}

One of possible approaches to the determination of crystal lattice type is offered in [Kup01a] where previously estimated lattice parameters are compared with predefined reference lattice parameters. The lattice is considered to belong to a particular type if its parameters have the closest match with the parameters of the reference lattice of this type.

Among basic lattice structure identification methods based on parameter estimation are:

- the comparator of the National Institute of Standards and Technology [Kes01a],

- packing efficiency-based identification (Fig. 4) [Smi01a],

- $\quad$ isosurface-based identification (Fig. 5) [Pat01a].

However, these approaches have some drawbacks that restrict their use: the tricky process of crystal preparation (the need for accurate polishing and mounting), low efficiency of comparison of similar lattices, high sensitivity to minor distortions of lattice node coordinates.

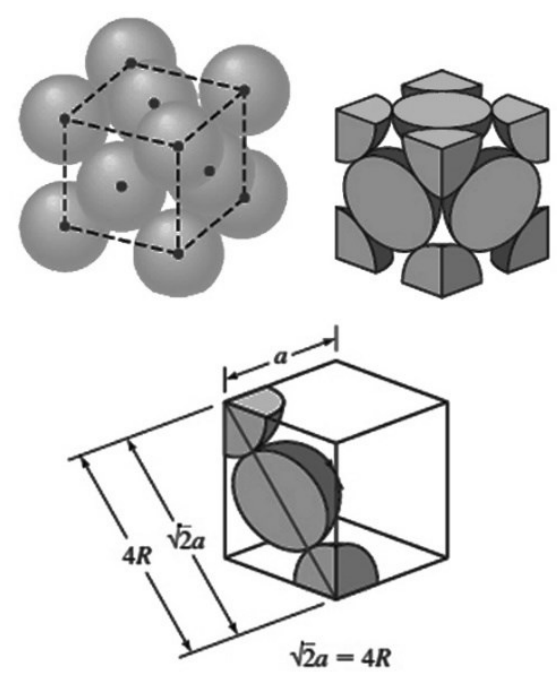

Figure 4. Close packing of spheres.
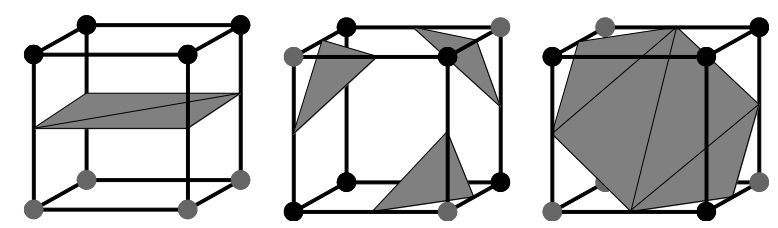

Figure 5. Types of isosurfaces constructed for a cubic lattice.

To overcome these drawbacks, we proposed a new algorithm for crystal lattice parametric identification based on the gradient steepest descent method [Shi01a]. In the algorithms, the result vectors of the lattice identification method based on estimation of Bravais unit cell parameters was used as the initial approximation. The main idea was to increase identification accuracy by the successive refinement of initial estimations (Fig. 6).

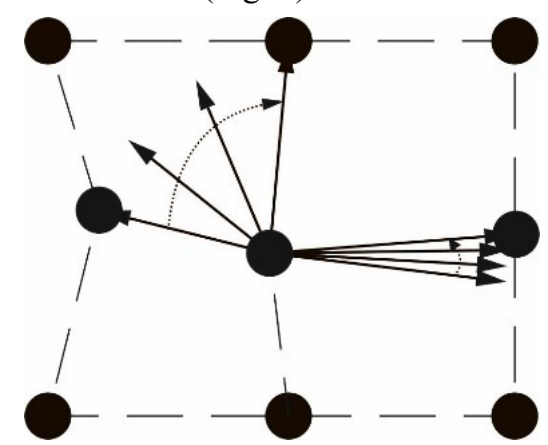

Figure 6. Refinement of translation vectors.

The proposed algorithm showed surprisingly high accuracy of parametric identification at the expense of high computational complexity. Nevertheless, the algorithm did not solve the problem of ambiguity of unit cell choice. In this paper, we offer a radically new approach based on fuzzy neural networks to solve the main problem of crystal lattice structural identification. 


\section{MODEL OF FUZZY NEURAL NETWORKS}

Figure 7 shows an example of fuzzy TSK multipleoutput neural network.

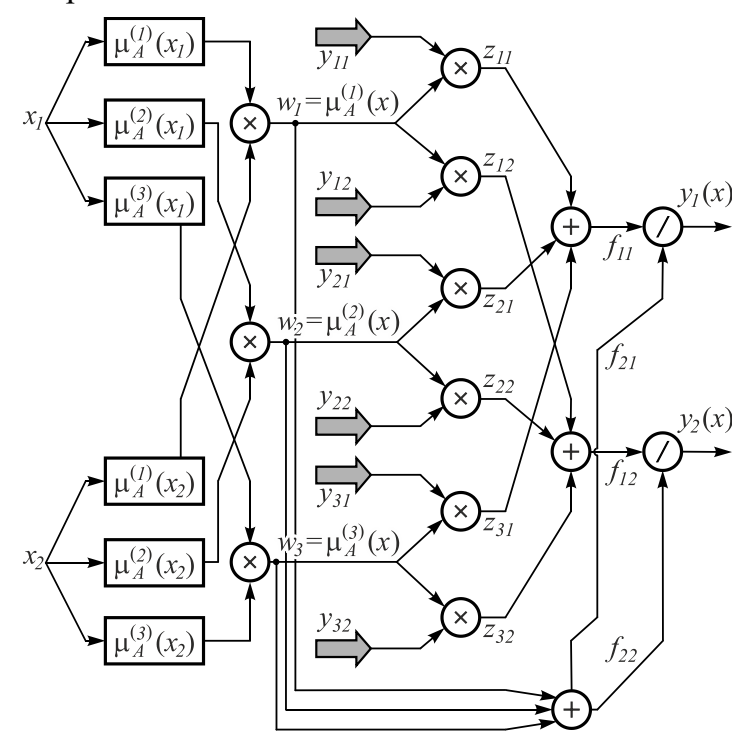

Figure 7. The structure of fuzzy TSK neural network with two inputs, three inference rules and two outputs.

Generalized Gauss function

$$
\mu_{A}\left(x_{j}\right)=\frac{1}{1+\left(\frac{x_{j}-c_{j}}{\sigma_{j}}\right)^{2 b_{j}}} .
$$

is used as a fuzzification function for each variable $x_{j}$

The fuzzy conjunction in the form of algebraic product

$$
\mu_{A}^{(i)}(x)=\prod_{j=1}^{N}\left[1 /\left(1+\left(\frac{x_{j}-c_{j}^{(i)}}{\sigma_{j}^{(i)}}\right)^{2 b_{j}^{(i)}}\right)\right]
$$

is used to aggregate the condition of the $i$-th rule.

Given $M$ inference rules, the aggregation of the network output is done by Equation 3, which can be represented as

$$
y(x)=\frac{1}{\sum_{i=1}^{M} w_{i}} \sum_{i=1}^{M} w_{i} y_{i}(x)
$$

where $y_{i}(x)=p_{i 0}+\sum_{j=1}^{N} p_{i j} x_{j}$ is the aggregation of implication. Weights $w_{i}$ in this expression are interpreted as components $\mu_{A}^{(i)}(x)$ defined by Equation 2.
The first layer of the network is responsible for fuzzification of each variable $x_{j}(j=1,2, \ldots, N)$ defining the coefficient of belonging $\mu_{A}^{(i)}\left(x_{j}\right)$ for each $i$-th inference rule according to the fuzzification function used. This is a parametric layer whose parameters $\left(c_{j}^{(i)}, \sigma_{j}^{(i)}, b_{j}^{(i)}\right)$ are subject to adaptation in learning.

The second layer makes aggregation of particular variables $x_{j}$ defining the resulting coefficient of belonging $w_{i}=\mu_{A}^{(i)}(x)$ in accordance with Equation 2. The third layer is the TSK function generator that calculates $y_{i}(x)=p_{i 0}+\sum_{j=1}^{N} p_{i j} x_{j}$. In addition, this layer computes the products of signals $y_{i}(x)$ and weights $w_{i}$ found in the previous layer. This is a parametric layer with adaptable linear weights $p_{i j}(i=1,2, \ldots, M ; j=1,2, \ldots, N)$.

The forth layer has two neuron-adders, one of which calculates the weighed sum of signals $y_{i}(x)$, and the other sums up the weights $\sum_{i=1}^{M} w_{i}$.

The fifth layer consists of several output neurons. This is a normalizing layer where the weights are normalized according to Equation 3. Output signals $y_{s}(x)$ are defined as

$$
y_{s}(x)=f_{s}(x)=\frac{f_{1 s}}{f_{2 s}}
$$

\section{THE LEARNING TECHNIQUE}

The data of generated unit cells of 7 different types were used for learning the neural network. The data were generated under the following conditions:

1. The number of lattices per each lattice system is 1000 .

2. The minimum admissible difference between "unequal" cell edges is 0.050 angst.

3. The minimum admissible difference between "unequal" cell angles is $0.020 \mathrm{rad}$.

4. The maximum admissible difference between the reference and estimated values of cell edges is 0.010 angst.

5. The maximum admissible difference between the reference and estimated values of cell angles is $0.010 \mathrm{rad}$.

The parameters of unit cell generation are: 
1. The minimum edge lengths are 1.000 angst, 1.000 angst, 1.000 angst.

2. The maximum edge lengths are 5.000 angst, 5.000 angst, 5.000 angst.

3. The minimum angle values $0.175 \mathrm{rad}, 0.175 \mathrm{rad}$, $0.175 \mathrm{rad}$.

4. The maximum angle values $1.571 \mathrm{rad}, 1.571 \mathrm{rad}$, $1.571 \mathrm{rad}$.
The size of lattice in each direction was taken equal to three nodes. The G6-space notation [And01a] was used to bring the parameters of unit cells to a common value range.

The preliminary examination of original data allowed us to divide 7 lattice types in 4 groups according to the quantity and ordinal numbers of non-zero columns in data files. The grouping of crystal lattices is given in Table 2.

\begin{tabular}{|l|c|c|c|c|c|c|c|}
\hline Lattice System Type & $l_{1}^{2}$ & $l_{2}^{2}$ & $l_{3}^{2}$ & $2 l_{2} l_{3} \cos \alpha_{1}$ & $2 l_{1} l_{3} \cos \alpha_{2}$ & $2 l_{1} l_{2} \cos \alpha_{3}$ & Subgroup No. \\
\hline Triclinic $(a P)$ & $\mathrm{x}$ & $\mathrm{x}$ & $\mathrm{x}$ & $\mathrm{x}$ & $\mathrm{x}$ & $\mathrm{x}$ & $\mathbf{1}$ \\
\hline Trigonal $(h R)$ & $\mathrm{x}$ & $\mathrm{x}$ & $\mathrm{x}$ & $\mathrm{x}$ & $\mathrm{x}$ & $\mathrm{x}$ & $\mathbf{1}$ \\
\hline Hexagonal $(h P)$ & $\mathrm{x}$ & $\mathrm{x}$ & $\mathrm{x}$ & $\mathrm{x}$ & 0 & 0 & $\mathbf{2}$ \\
\hline Monoclinic $(m P)$ & $\mathrm{x}$ & $\mathrm{x}$ & $\mathrm{x}$ & 0 & 0 & $\mathrm{x}$ & $\mathbf{3}$ \\
\hline Orthorhombic $(o P)$ & $\mathrm{x}$ & $\mathrm{x}$ & $\mathrm{x}$ & 0 & 0 & 0 & $\mathbf{4}$ \\
\hline Tetragonal $(t P)$ & $\mathrm{x}$ & $\mathrm{x}$ & $\mathrm{x}$ & 0 & 0 & 0 & $\mathbf{4}$ \\
\hline Cubic $(c P)$ & $\mathrm{x}$ & $\mathrm{x}$ & $\mathrm{x}$ & 0 & 0 & 0 & $\mathbf{4}$ \\
\hline
\end{tabular}

Table 2. Grouping of lattice system types.

After that the TSK neural network was subjected to learning and tested in three stages:

1. Pair training and testing of the neural network for recognition of 2 lattice types;

2. Training and testing of the neural network for recognition of all 7 lattice types;

3. Training and testing of the neural network for recognition of lattice types in subgroups 1 and 4 .

\section{DETERMINING THE CRYSTAL LATTICE TYPE}

The relative error of structure identification in all experiments was calculated as a percentage of identification failures over the whole test lattice collection. At the first stage 6-dimensional vectors comprising of learning data of two types were fed to the TSK neural net. The output layer held two neurons according to the number of classes being recognized. The results are shown in Table 3.

It is worth noticing that the neural net could not discriminate triclinic lattices (in fact, arbitrary lattices) from trigonal lattices (three equal edges and three equal angles). The reason is that the placing of these two lattice types in a single subgroup is not entirely correct: triclinic lattices are described by six independent parameters (six non-zero columns), and trigonal lattices by two independent parameters (also six non-zero columns). So, we put these two lattice types in one subgroup "formally" rather than "physically".
At the second stage of the investigation, the data collection presenting all the seven lattice types was used to train the neural net. Six-dimensional vectors made up of this data were fed to the TSK neural net. According to the number of classes to be recognized, the output layer had seven neurons. The experimental results show that the network recognize hexagonaland monoclinic-type lattices (subgroups 2 and 3 ) without failure. It is because the learning data for these lattice types has different combinations of zero and non-zero columns than that for other lattice types. In other words, the neural net recognize the lattices of hexagonal and monoclinic type as non-overlapping classes.

\begin{tabular}{|l|c|c|c|c|c|c|}
\hline & $\boldsymbol{h R}$ & $\boldsymbol{h P}$ & $\boldsymbol{m P}$ & $\boldsymbol{o P}$ & $\boldsymbol{t P}$ & $\boldsymbol{c P}$ \\
\hline $\boldsymbol{a P}$ & 10 & 0 & 0 & 0 & 1 & 1 \\
\hline $\boldsymbol{h R}$ & & 0 & 0 & 0 & 0 & 2 \\
\hline $\boldsymbol{h P}$ & & & 15 & 15 & 43 & 12 \\
\hline $\boldsymbol{m P}$ & & & & 42 & 16 & 10 \\
\hline $\boldsymbol{o P}$ & & & & & 16 & 8 \\
\hline $\boldsymbol{t P}$ & & & & & & 12 \\
\hline
\end{tabular}

Table 3. Relative errors of crystal lattice structure identification in pair learning of the TSK network using a 7000-lattice sample.

Additionally, the third stage of experiments was carried out to recognize lattice types belonging to 
subgroups 1 and 4. The TSK neural net with 6 inputs and 2 outputs were used to deal with lattices of subgroup 1. The same net with 3 inputs corresponding to non-zero columns of initial data and 3 outputs were engaged to process subgroup 4. The identification failure rate of the TSK neural net was $10 \%$ for subgroup 1, and $25 \%$ for subgroup 4.

Let us compare the values of the relative errors with the results presented in [Kir01a, Kup01a] where the recognition of lattice types was done with the aid of parametric identification methods. By way of example let us look at the best result of structure identification obtained in comparative estimation of Bravais cell parameters and Wigner-Seitz cell volumes [Kup01a] (see Table 4).

\begin{tabular}{|l|c|c|c|c|c|c|}
\hline & $\boldsymbol{h R}$ & $\boldsymbol{h P}$ & $\boldsymbol{m P}$ & $\boldsymbol{o P}$ & $\boldsymbol{t P}$ & $\boldsymbol{c P}$ \\
\hline $\boldsymbol{a P}$ & 0 & 0 & 1 & 0 & 0 & 0 \\
\hline $\boldsymbol{h R}$ & & 0 & 0 & 2 & 3 & 26 \\
\hline $\boldsymbol{h P}$ & & & 7 & 0 & 0 & 0 \\
\hline $\boldsymbol{m P}$ & & & & 22 & 10 & 0 \\
\hline $\boldsymbol{o P}$ & & & & & 34 & 15 \\
\hline $\boldsymbol{t P}$ & & & & & & 26 \\
\hline
\end{tabular}

Table 4. Relative errors of crystal lattice structure identification using parametric identification methods.

The comparison shows that the use of neural nets makes it possible to significantly decrease the structure identification failure rates for the following lattice types:

- trigonal and cubic types from 26 to $2 \%$;

- orthorhombic and tetragonal types from 34 to $16 \%$;

- tetragonal and cubic types from 26 to $12 \%$;

- orthorhombic and cubic types from 15 to $8 \%$.

On the other hand, when discriminating monoclinic and hexagonal lattices from lattices of subgroups 3 and 4 , the neural net gives much worse results than parametric identification methods. Particularly, in separation of hexagonal lattices from tetragonal ones the relative error has grown from 0 to $43 \%$.

As for subgroup 4, here the low results are due to the geometric overlapping of classes. A set of cubic-type lattices (red diagonal in Figure 8) lie in the same line in the three-dimensional space. This line is in the plane containing tetragonal-type elements (the dark-grey layer in Figure 8). The plane lies in turn inside the parallelogram formed by orthorhombic-type elements (the light-grey cube in Figure 8).

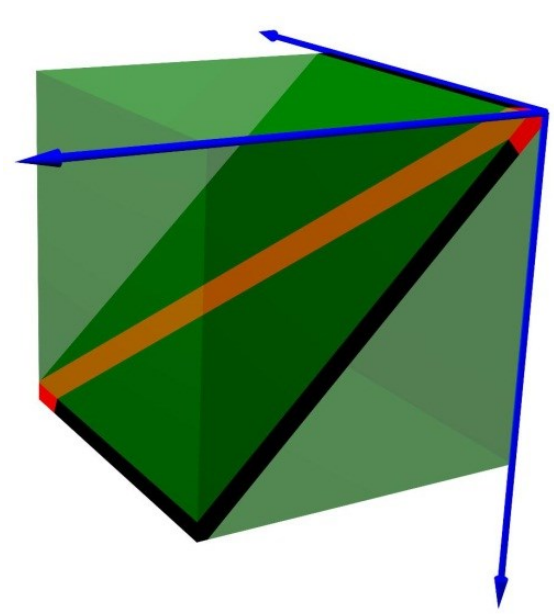

Figure 8. The class overlapping of lattice types of subgroup 4.

\section{CONCLUSION}

We have offered a three-stage learning technique for neural networks. Crystal lattices are divided into nonoverlapping classes in the first two stages. Crystal lattices belonging to overlapping classes are recognized at the last stage.

As compared with parametric identification methods, the use of neural nets makes it possible to decrease the 3D structure identification failure rate for four couples of lattice systems considerably (as much as 2 to 13 times).

The research results allow us to draw a conclusion that fuzzy neural networks are an efficient tool in recognition of crystal lattice types using Bravais cells parameters.

\section{ACKNOWLEDGEMENTS}

This work was partially supported by the Ministry of education and science of the Russian Federation in the framework of the implementation of the Program of increasing the competitiveness of Samara University among the world's leading scientific and educational centers for 2013-2020 years; by the Russian Foundation for Basic Research grants (\# 15-29-03823, \# 16-41-630761, \# 17-01-00972, \# 18-37-00418); in the framework of the state task \#0026-2018-0102 "Optoinformation technologies for obtaining and processing hyperspectral data".

\section{REFERENCES}

[And01a] Andrews, L.C., Bernstein, H.J., Lattices and reduced cells as points in 6-space and selection of Bravais lattice type by projections. Foundations of crystallography, 44(6), pp.10091018, 1988

[Ham01a] Hammond, C., The basic of crystallography and diffraction, 3rd ed. Oxford University Press, pp.55-83, 2009 
[Kat01a] Katasyov, S.A., Software for Building Fuzzy Rule Inference Data Bases for Expert Systems. Fundamental Research, 10(9), pp.1922-1927, 2013

[Kes01a] Kessler, E.G., Henins, A., Deslattes, R.D., Nielsen, L., Arif M., Precision comparison of the lattice parameters of silicon monocrystals. Journal of research of the national institute of standards and technology. 99(1), pp.1-18, 1994

[Kip01a] Kiper, A.V., Stankevich, T.S., Development of a Fuzzy Classifier Using the Sugeno Fuzzy System for Ranking a Fire on the Seaport Premises. Astrakhan State Technical University Bulletin, Marine Engineering Series, 2, pp.18-25, 2012

[Kir01a] Kirsh, D.V., Kupriyanov, A.V., Parallel implementations of parametric identification algorithms for three-dimensional crystal lattices. CEUR Workshop Proceedings, 1638, pp.451459,2016

[Kup01a] Kupriyanov, A.V., Kirsh, D.V., Estimation of the Crystal Lattice Similarity Measure by Three-Dimensional Coordinates of Lattice Nodes. Optical Memory \& Neural Networks (Information Optics), 24(2), pp. 145151,2015

[Nov01a] Novak, V., Perfilieva, I., Mockor, J., Mathematical principles of fuzzy logic. The Kluwer International Series in Engineering and Computer Science, 517, 352 pages, 1999

[Oso01a] Osovsky, S., Data Processing Neural Nets. Finance and Statistics, 344 pages, 2002
[Pat01a] Patera, J., Skala, V., Centered cubic lattice method comparison. Proceedings of algoritmy, pp.309-319, 2005

[Rut01a] Rutkovskaya, D., Pilinsky, M., Rutkovsky, L., Neural Nets, Genetic Algorithms and Fuzzy Systems. Hotline-Telecom, 52 pages, 2006

[Shi01a] Shirokanev, A.S., Kirsh, D.V., Kupriyanov, A.V., Development of the crystal lattice parameter identification method based on the gradient steepest descent method. 24 ${ }^{\text {th }}$ International Conference in Central Europe on Computer Graphics, Visualization and Computer Vision WSCG 2016, CSRN 2603, pp. 65-68, 2016

[Smi01a] Smith, W.F., Foundations of Materials Science and Engineering. McGraw-Hill, pp.67107,2004

[Sol01a] Soldatova, O.P., Lyozin, I.A., Solving the Classification Problem Using Mamdani-Zadeh System-Based Fuzzy Neural Rule Inference Networks. Samara Research Center Bulletin. Physical and Mathematical Sciences Series, 16(2), pp.136-148, 2014

[Til01a] Tilley, R., Crystals and crystal structure. John Wiley \& Sons, pp.17-32, 2006

[Vin01a] Vineetha, S., Bhat, C.C.S., Idicula, S.M., MicroRNA-mRNA interaction network using TSK-type recurrent neural fuzzy network. Gene, 515(2), pp.385-390, 2013 